\title{
Performance Analysis of Antenna Selection Techniques in MIMO-OFDM System with Hardware Impairments: Energy Efficiency perspective
}

\author{
Anuj Singal, Deepak Kedia \\ Department of Electronics and Communication Engineering' GJU S\&T, Hisar (HARYANA), India
}

\begin{tabular}{l} 
Article Info \\
\hline Article history: \\
Received Oct 12, 2017 \\
Revised Jan 5, 2018 \\
Accepted Jun 10, 2018 \\
\hline Keyword: \\
Antenna selection (AS), \\
Energy Efficiency (EE), \\
Multiple-input multiple output \\
(MIMO), \\
Orthogonal-frequency division \\
multiplexing (OFDM)
\end{tabular}

\section{Article Info}

Revised Jan 5, 2018

\begin{abstract}
In this paper we propose a new MIMO-OFDM model in which we consider various antenna selection techniques like Bulk selection and Per-subcarrier selection etc. with hardware impairments such as non-linearties of amplifiers, quantization noise, phase noise and I-Q imbalance etc. As we know that the transceiver hardware impairments limit the channel capacity and the energy efficiency of MIMO-OFDM system, so we can not neglect the fundamental impacts of these hardware impairments $\{$ Kappa $\in(0.050 .1)\}$ on the energy efficiency in the high SNR domain. Therefore we analyze the Energy Efficiency of Bulk and Per-subcarrier antenna selection techniques with or without hardware impairments. It has been observed that the energy efficiency decreases as the value of these hardware impairments increases. As we compared the Bulk antenna selection with the Per-subcarrier antenna selection scheme, the Per-subcarrier antenna selection requires more number of RF (radio frequency) chains and transmits power in comparison to the Bulk selection. Due to this, the Bulk antenna selection technique is more energy efficient than Per-subcarrier antenna selection.
\end{abstract}

Copyright $\odot 2018$ Institute of Advanced Engineering and Science. All rights reserved.

\section{Corresponding Author:}

Anuj Singal,

Department of Electronics and Communication Engineering,

GJU S\&T, Hisar (HARYANA), India.

Email: anuj.singal08@gmail.com

\section{INTRODUCTION}

In recent years, the demands of high speed wireless communication have been increasing steadily. To meet the increasing demand it requires to use the large number of transmit and receive antenna i.e. MIMO. The MIMO is further integrated with OFDM to improve the spectral efficiency (SE) or the energy efficiency (EE). The spectral efficiency is one of the main performance parameters or indicators for the efficient designing and optimizing the wireless communication system. But the use of large number of transmit and receive antennas in MIMO-OFDM system will lead to increased consumption of energy, since it requires the extra complex circuit like multiple RF chains and more complex signal processing at both the transmitter and receiver side. Therefore, energy consumption is one of the main critical problems and has become a global concern for the industrial and academic researcher [1].

The energy efficiency (EE), the ratio of number of transmitted information (bits) and the energy (bits/Joule), is one of the most significant design key metrics for the future wireless networks. The energy efficiency of a wireless link depends on many factors like SNR, transceiver hardware impairments, channel estimation accuracy and the signal processing resource. A lot of researchers are working on MIMO-OFDM with various antenna selection techniques but the impact of transceiver hardware impairments (amplifier nonlinearities, carrier-frequency, quantization noise, IQ-imbalance, sampling-rate jitter/offsets and phase noise, etc.) has not been explored much so far [2]. 
Today, Antenna selection techniques are used in conventional MIMO-OFDM systems for reducing the system complexity. In this technique, a subset of all available antennas are chosen for transmitting or receiving the signal which help in reducing the resource requirement and hardware complexity of MIMOOFDM systems. So, MIMO-OFDM systems with Antenna selection perform significantly better than without antenna selection process [3]. A sub-optimal transmit antenna selection techniques has been studied for maximizing the capacity of the channel. Also, the shape of the channel correlation matrices examined and provides the solution of the spectral decomposition problem $[4,5]$.

A low complexity antenna subset-selection technique has been proposed for green relaying MIMO wireless communications system. Also, the transmit power and number of active antennas has been jointly optimized by using both the maximum Frobeninus norm and adaptive beam forming [6]. In an EnergyEfficient model a trade-off between power consumption and data rate has been explored by using various antenna selection schemes [7]. In [8], the combination idea of both constructive inter-channel interference and antenna selection are analyzed for improving the efficiency in Massive-MIMO system. The multiplexing gain and capacity limit of MIMO system are analyzed in transceiver hardware impairments environments [9].

\subsection{Problem Statement}

The extensive literature survey revealed that the consideration of impact of hardware impairments in Antenna selection may lead to improved analysis of performance of MIMO-OFDM system. So, in this paper we propose a new MIMO-OFDM model in which we consider various antenna selection techniques like Bulk selection and Per-subcarrier selection etc. along with hardware impairments. The main contribution of this paper is summarized below:

a. A new Energy Efficiency equation has been derived with hardware impairments using antenna selection schemes like Per-subcarrier selection and Bulk selection.

b. In the proposed model, the Energy Efficiency of various antenna selection schemes like Bulk, Persubcarrier selection is analyzed by varying the transmit power, number of transmitting and receiving antenna, levels of hardware impairments and the types of antenna selection.

The remainder of this paper has been organized as follows. Section 2 gives an overview of MIMOOFDM system with antenna selection. The various antenna selection techniques are reviewed in Section 3. Section 4 includes research method in which we explains the energy efficiency for various antenna selection techniques in brief. The simulation results are discussed in the section 5 and finally conclusions are drawn in section 6 .

\section{MIMO-OFDM System with Antenna Selection}

In this section, a transmitter and receiver block diagram of MIMO-OFDM system with antenna selection using $\mathrm{n}_{\mathrm{T}}$ transmit and $\mathrm{n}_{\mathrm{R}}$ receive antenna is shown in Figure 1 and 2 respectively. In the transmitter part, firstly input data is processed by the MIMO-OFDM system by using the transmit antenna selection techniques. In transmit and receive antenna selection part, RF switching unit helps to select the number of RF chains. In the end MIMO-OFDM processing is used to recover the transmit data as shown in Figure 2. As we know that the MIMO is used to increase the capacity of the system, these multiple radio frequency (RF) chains increase the system complexity, cost and the power consumption. So, antenna selection is used to overcome this problem at transmitter and receiver side by selecting the subset of best transmit and receive antenna among the available antenna [10]. There exist various antenna selection techniques like per-subcarrier or bulk selection and the brief introduction of these schemes is presented in the next section.

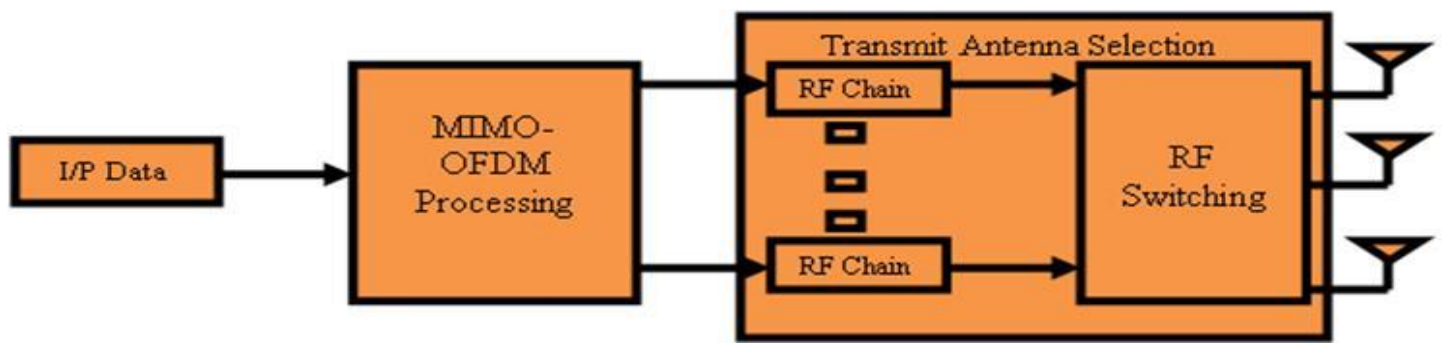

Figure 1. MIMO-OFDM Transmitter with Antenna Selection 


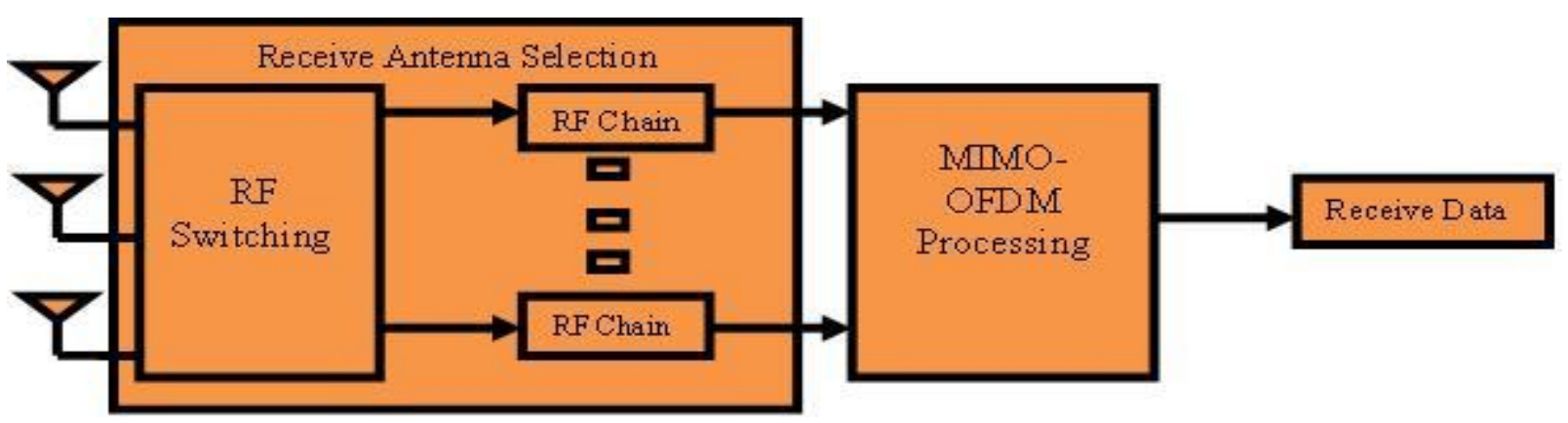

Figure 2. MIMO-OFDM Receiver with Antenna Selection

\section{ANTENNA SELECTION TECHNIQUES}

The brief introduction of the two fundamental antenna selection schemes considered in this paper i.e. per-subcarrier or bulk selection is presented below:

a. Bulk selection $[10,11]$ : - In this scheme, we select only one antenna which has large accumulated-cost among all available transmitting antennas $n_{T}$. Also, it requires the smallest number of RF chain as compared to other selection approaches. Further, the total power consumed for the one MIMO-OFDM symbol is given in equation 1 below [10]

$$
\mathrm{P}_{\text {total }}=\mathrm{n}_{\mathrm{on}}\left(\mathrm{P}_{\mathrm{PA}}+\mathrm{P}_{\mathrm{ctx}}\right)+\mathrm{n}_{\mathrm{R}} \mathrm{P}_{\mathrm{crx}}+\mathrm{P}_{\mathrm{bb}}
$$

In the above equation, the number of active Radio Frequency (RF) chains is equal to the $\mathrm{n}_{\mathrm{on}}$, $\mathrm{P}_{\mathrm{PA}}$ represents the power consumed by one power amplifier, $\mathrm{P}_{\mathrm{ctx}}$ and $\mathrm{P}_{\mathrm{crx}}$ are the power consumed by one transmitting and receiving branch respectively, $\mathrm{n}_{\mathrm{R}}$ is the number of receiving antenna in the system and $\mathrm{P}_{\mathrm{bb}}$ is the sum of power consumed by the various baseband processing units existing at the transmitter and receiver. $\mathrm{P}_{\mathrm{PA}}$ is the dc power drawn and it can be expressed as shown in equation 2 below [10]

$$
P_{P A}=\frac{P_{t}\left(\frac{K}{n_{o n}}\right)}{\eta}=\frac{\frac{P_{T}}{n_{\mathrm{on}}}}{\eta}
$$

In this equation $K$ is the number of subcarriers, $P_{t}$ is the transmit power given to the each subcarrier, $\eta$ is the efficiency of power amplifier and $\mathrm{K} / \mathrm{n}_{\mathrm{on}}$ is the number of symbols allocated to each transmit antenna. If we put the $\mathrm{P}_{\mathrm{PA}}$ as shown in equation 2 in the equation 1 then we can represents the total power consumption $\left(\mathrm{P}_{\text {total }}\right)$ equivalent to the equation $3[10]$ as shown below:

$$
\mathrm{P}_{\text {total }}=\frac{\mathrm{P}_{\mathrm{T}}}{\eta}+\mathrm{n}_{\mathrm{on}} \mathrm{P}_{\mathrm{ctx}}+\mathrm{n}_{\mathrm{R}} \mathrm{P}_{\mathrm{crx}}+\mathrm{P}_{\mathrm{bb}}
$$

In Bulk antenna selection scheme the number of active Radio Frequency $(R F)$ chains $\left(n_{o n}\right)$ are equal to the one, therefore the total power consumption in Bulk antenna selection scheme as shown below [10] in equation 4:

$$
\mathrm{P}_{\text {total }}^{\text {Bulk }}=\frac{\mathrm{P}_{\mathrm{T}}}{\eta}+\mathrm{P}_{\mathrm{ctx}}+\mathrm{n}_{\mathrm{R}} \mathrm{P}_{\mathrm{crx}}+\mathrm{P}_{\mathrm{bb}}
$$

b. Per-sub carrier selection [10]: - In per-sub carrier selection, antenna selection is independent for each sub-carrier as compared with the bulk selection. Due to this, it requires more number of RF chains and provides better error performance. So, the number of active Radio Frequency $(R F)$ chains $\left(n_{o n}\right)$ required is equal to the transmitting antennas $n_{T}$. Therefore, the total power consumption in per subcarrier antenna selection scheme is shown below [10] in equation 5 :

$$
\mathrm{P}_{\text {total }}^{\text {per }}=\frac{\mathrm{P}_{\mathrm{T}}}{\eta}+\mathrm{n}_{\mathrm{T}} \mathrm{P}_{\mathrm{ctx}}+\mathrm{n}_{\mathrm{R}} \mathrm{P}_{\mathrm{crx}}+\mathrm{P}_{\mathrm{bb}}
$$


As we know that the transceiver hardware impairments limits the MIMO-OFDM channel capacity and the energy efficiency, therefore the energy efficiency for the above selection techniques with or without hardware impairments is being analyzed in the next section.

\section{Research Method: Energy Efficiency of the Per-Subcarrier and the Bulk Antenna Selection Technique}

A brief analysis of the energy efficiency and its value for the Bulk and per subcarrier selection technique with or without hardware impairments is presented below:

\subsection{Energy Efficiency [11]}

Energy efficiency is defined as the ratio between the information rate that is equivalent to capacity of the system and total power consumed in the system as shown in equation 6 below [10]:

$$
\text { Energy Efficiency }(\mathrm{EE})=\frac{\text { Capacity }(\mathrm{C})}{\text { Total Power Consumption }\left(\mathrm{P}_{\mathrm{T}}\right)}
$$

Here, $\mathrm{P}_{\mathrm{T}}$ is the total power consumption and its value for the Bulk and Per-Subcarrier selection technique is given by equation 4 and 5 respectively. Also, the Channel capacity (C) is defined as the maximum possible transmission information rate with a minimum error that can be supported by the channel. The channel capacity can be divided into two parts in which first part will be the ideal one and latter one considers the hardware impairments. So the channel capacity with and without hardware impairments is explained below:

a. Channel capacity without hardware impairments [12, 13]: The channel capacity without hardware impairments (conventional MIMO-OFDM system) has a high SNR slope which is equal to the minimum of the number of transmit and receive antennas. It's means the channel capacity of conventional system increases unboundedly as we increases the number of transmit and receive antenna and SNR as shown in Figure 3 in the next section. It has been observed that the channel state information (CSI) is improved the channel capacity of MIMO-OFDM system and it is obtained by using the pilot signal at the transmitter. But it is a very costly and challenging problem. So, today the impact of CSI in MIMO-OFDM system is a very hot topic for the research in wireless system [14]. The ideal channel capacity of MIMO/ MIMOOFDM system with or without channel information are summarized in the Table 1 below:

Table 1. Ideal Channel Capacity of MIMO/MIMO-OFDM system with or without CSI [12, 13]

\begin{tabular}{lll}
\hline \hline Sr. No. & Channel & Channel Capacity \\
\hline 1. & MIMO without CSI & $E\left\{\sum_{r=1}^{R} \log _{2}\left(1+\frac{\rho}{n_{T}} \lambda_{r}\right)\right\}(\mathrm{bit} / \mathrm{sec} / \mathrm{Hz})$ \\
2 & MIMO with CSI & $E\left\{\sum_{r=1}^{R} \log _{2}\left(1+\Psi_{r} \frac{\rho}{n_{T}} \lambda_{r}\right)\right\}(\mathrm{bit} / \mathrm{sec} / \mathrm{Hz})$ \\
3 & MIMO-OFDM without CSI & $E\left\{\frac{1}{K} \sum_{k=0}^{K-1} \log _{2} \operatorname{det}\left(I_{n_{R}}+\frac{\rho}{n_{T}} H(k) H^{H}(k)\right)\right\}(\mathrm{bit} / \mathrm{sec} / \mathrm{Hz})$ \\
4 & MIMO-OFDM with CSI & $E\left\{\frac{1}{K} \sum_{k=0}^{K-1} \log _{2} \operatorname{det}\left(I_{n_{R}}+\Psi_{r} \frac{\rho}{n_{T}} H(k) H^{H}(k)\right)\right\}(\mathrm{bit} / \mathrm{sec} / \mathrm{Hz})$ \\
\hline
\end{tabular}

Here $\mathrm{E}\{$.$\} is the expectation vector, \mathrm{R}$ is the rank of channel matrix, $n_{T}$ is the number of transmit antenna, $\rho$ is the signal to noise ratio, $\lambda_{r}$ is the $\mathrm{r}^{\text {th }}$ eigen value, $\mathrm{K}$ is the number of sub-carriers and $\psi_{r}$ is the optimal transmit power factor.

b. Channel capacity with hardware impairments $[15,16]$ : The channel capacity without hardware impairments increases unboundedly with increase in no. of antennas and SNR as explained above. But the hardware impairments (amplifier non-linearties, carrier-frequency, quantization noise, IQ-imbalance, sampling-rate jitter/offsets and phase noise, etc.) limit the channel capacity as shown in Figure 4 in the next section. This capacity limit depends on level of impairments and as we increase the level of impairments, the channel capacity is decreased. So, the channel capacity with hardware impairments is shown below in equation 7 [15] :

$$
\mathrm{C}_{\mathrm{N}_{\mathrm{t}} \mathrm{N}_{\mathrm{r}}}(\mathrm{SNR})=\operatorname{Sup}_{\mathrm{Q}: \operatorname{tr}(\mathrm{Q})=1} \mathrm{E}_{\mathrm{H}}\left\{\log _{2} \operatorname{det}\left(\mathrm{I}+\frac{\mathrm{SNRHQH}}{\mathrm{SNRHY}_{\mathrm{t}} \mathrm{H}^{\mathrm{H}}+\mathrm{I}}\right)\right\}
$$


Here $\mathrm{Q}=\mathrm{E}\left\{\mathrm{xx}^{\mathrm{H}}\right\}$ and $\mathrm{x}$ is the transmitted signal. In this we assumed that the normalized power constraint i.e. trace of $\mathrm{Q}\{\operatorname{tr}(\mathrm{Q})\}$ is equal to $1 . \mathrm{H}$ is the deterministic channel. Also $\Upsilon_{\mathrm{t}}=\operatorname{diag}\left(v_{1}\left(q_{1}\right) \ldots v_{N_{T}}\left(q_{N_{t}}\right), v_{n}\left(q_{n}\right)\right.$ is the variance, $N_{t}$ is the $\mathrm{n}^{\text {th }}$ transmitter and $q_{n}$ is the signal power. If we assume $\mathrm{Q}=\frac{1}{N_{\mathrm{t}}} \mathrm{I}$ then $\Upsilon_{\mathrm{t}}$ become equal to $\frac{k^{2}}{N_{t}} I$. In $\frac{k^{2}}{N_{t}} I, k$ (kappa) represents the hardware impairments and its range is from 0.05 to 0.1 [16]. If we decompose $\mathrm{H}$ into Eigen value, then above capacity equation 7 becomes equivalent to the following equation 8 [15]:

$$
\mathrm{C}_{\mathrm{N}_{\mathrm{t}} \mathrm{N}_{\mathrm{r}}}(\mathrm{SNR})=\sum_{\mathrm{i}=1}^{\mathrm{M}} \log _{2}\left(1+\frac{\operatorname{SNR} \lambda_{\mathrm{i}} \mathrm{d}_{\mathrm{i}}}{\operatorname{SNR} \lambda_{\mathrm{i}} \frac{\mathrm{k}^{2}}{\mathrm{~N}_{\mathrm{t}}}+1}\right)
$$

Here, $\mathrm{M}$ is the multiplexing gain, $\lambda_{\mathrm{i}}$ is the $\mathrm{i}^{\text {th }}$ Eigen value, $d_{i}=\left[\mu-\frac{1}{\lambda_{i}}\right]$ and $\mu$ is selected such that $\sum_{i=1}^{M} d_{i}=1$. The value of the energy efficiency for the Bulk and Per-Subcarrier antenna selection technique can be calculated by putting the value of the total power consumption for the Bulk and per subcarrier selection technique given by equation 4 and 5 respectively and the capacity with hardware impairments given by equation 8 in the equation 6 . Thus, the proposed energy efficiency of the Bulk and PerSubcarrier antenna selection technique in MIMO-OFDM System is summarized in the Table 2 below:

Table 2. Proposed Energy Efficiency of the Bulk and Per-Subcarrier Antenna Selection Technique in MIMOOFDM System

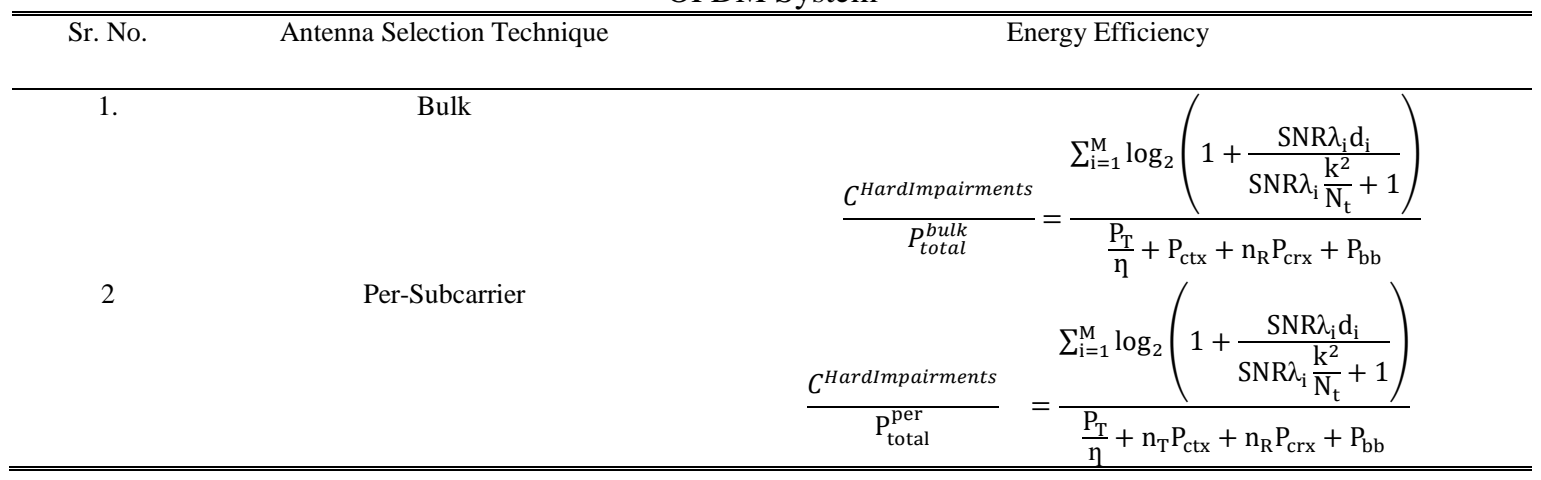

\section{RESULTS AND DISCUSSIONS}

In this section, we examined the impact of hardware impairments in various Antenna selection schemes which was not analyzed in the literature. So, energy efficiency of MIMO-OFDM channel is compared with respect to hardware impairments, SNR, transmit power and number of transmit and receive antennas for various antenna selection schemes like Bulk selection and Per subcarrier selection from Figure 3 to Figure 7 respectively.

\subsection{Energy Efficiency v/s Transmit Power}

In Figure 3 we consider Bulk and Per sub carrier antenna selection techniques for $4 \times 4$ transmit and receive antenna with or without contemplation of hardware impairments. Figure 3 shows that the energy efficiency decreases exponentially for the low transmit power consumption region $\{0.1$ to 0.4 watt $\}$. But the effect of transmit power consumption on the energy efficiency is very less in the higher transmit power consumption region $\{1.2$ to 1.8 watt $\}$. As we compared the energy efficiency of both antenna selection techniques then it is observed that the Bulk antenna selection has more energy efficiency than per subcarrier antenna selection. Also, the energy efficiency decreases as the level of the hardware impairments increases. Figure 4 shows the energy efficiency versus transmit power for various number of transmit and receive antennas from $2 \mathrm{X} 2$ to $5 \mathrm{X} 5$ with hardware impairments 0.1 It has been observed that the energy efficiency increases with increase in the number of transmit and receive antennas. 


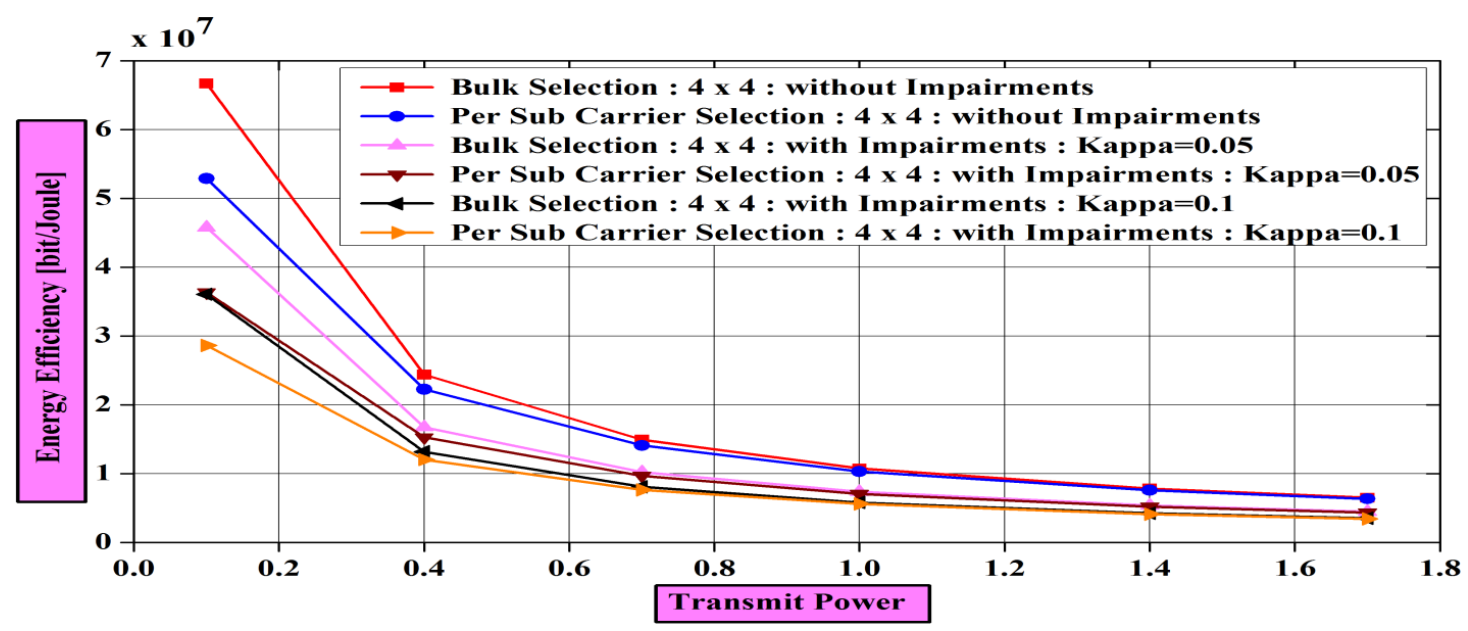

Figure 3. Energy Efficiency of MIMO-OFDM channel v/s transmit power for Bulk and Per-subcarrier antenna selection techniques with or without hardware impairments (Kappa=0.05, 0.1).

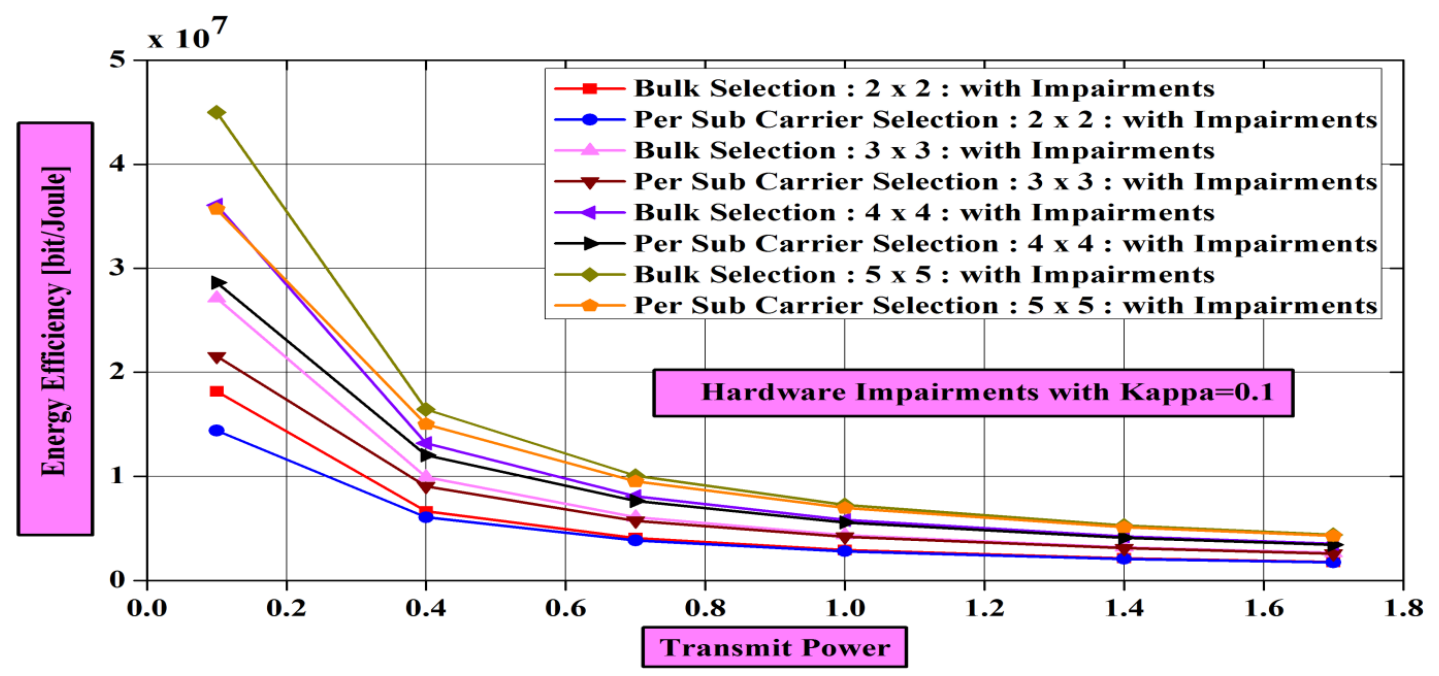

Figure 4. Energy Efficiency of MIMO-OFDM channel v/s transmit power for Bulk and Per-subcarrier antenna selection techniques using various transmit and receive antennas schemes $(2 \times 2,3 \times 3,4 \times 4,5 \times 5)$ with hardware impairments (Kappa=0.1).

\subsection{Energy Efficiency v/s SNR}

In Figure 5 the energy efficiency is compared with respect to SNR using various transmit powers $(\mathrm{P}=0.1,0.13,0.16,0.19,0.22,0.25$ watt $)$ for $4 \mathrm{X} 4$ transmit and receive antenna. Figure 5 shows that the Bulk antenna selection has more energy efficiency than per subcarrier antenna selection and the energy efficiency decreases with increase in the value of transmit power consumption. Also, the energy efficiency increases unboundedly as the SNR value increases. But this energy efficiency is limited by hardware impairments in the high SNR region (40 to $70 \mathrm{~dB}$ ) as shown in Figure 6. Also, Figure 6 shows that the energy efficiency decreases as the level of hardware impairment increases for 6x6 transeiver antenna system. Further, In Figure 7 we compared the energy efficiency with respect to SNR using various number of transmit and receive antenna schemes $(2 \times 2,4 \times 4,6 \times 6,8 \times 8)$ for the hardware impairments 0.1 . In this, the energy efficiency increases with increase in the number of transmit and receive antenna and it increases upto $80 \%$ as we compared 2x2 with $8 \times 8$ transeiver antenna scheme. Also, Bulk antenna selection has more energy efficiency than per subcarrier antenna selection in the high SNR region (20 to $70 \mathrm{~dB})$. But the energy efficiency remain almost same for both antenna selection techniques in the low SNR region (-10 to $20 \mathrm{~dB})$. 


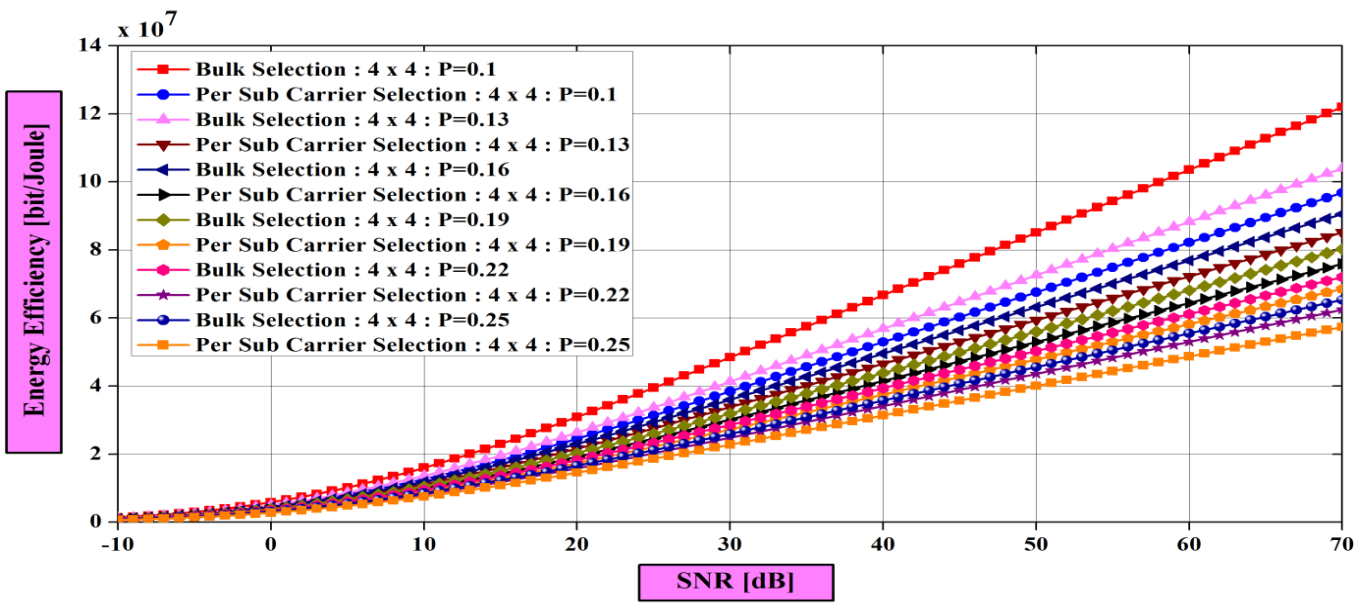

Figure 5. Energy Efficiency of MIMO-OFDM channel v/s SNR for Bulk and Per-subcarrier antenna selection techniques using various transmit power $(\mathrm{P}=0.1,0.13,0.16,0.19,0.22,0.25)$

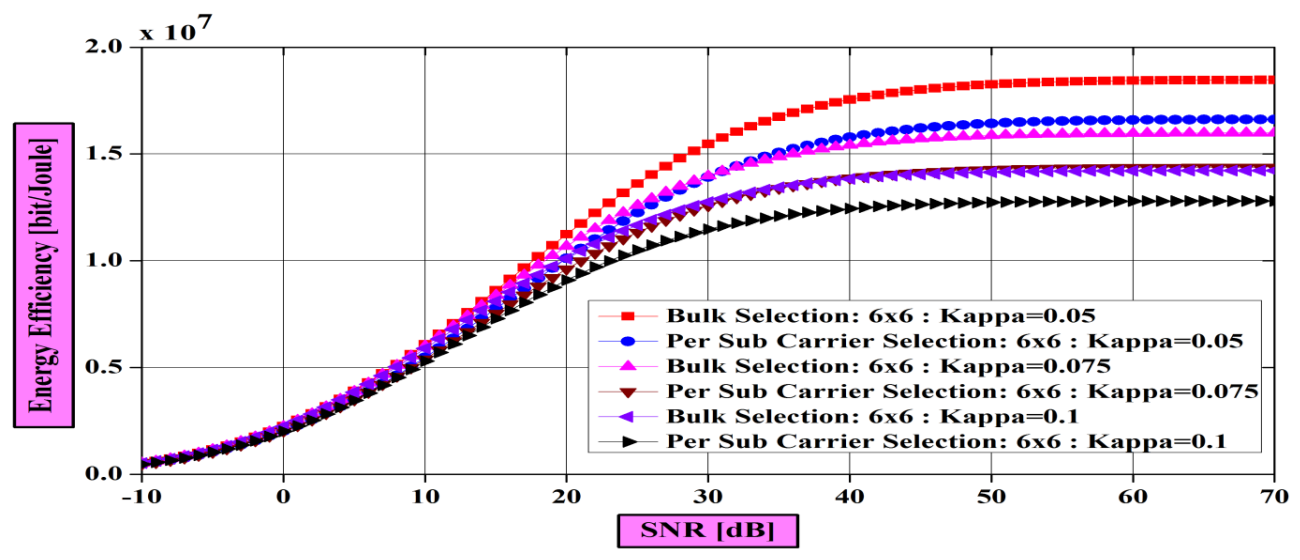

Figure 6. Energy Efficiency of MIMO-OFDM channel v/s SNR for Bulk and Per-subcarrier antenna selection techniques with various hardware impairments (Kappa=0.05, 0.075, 0.1).

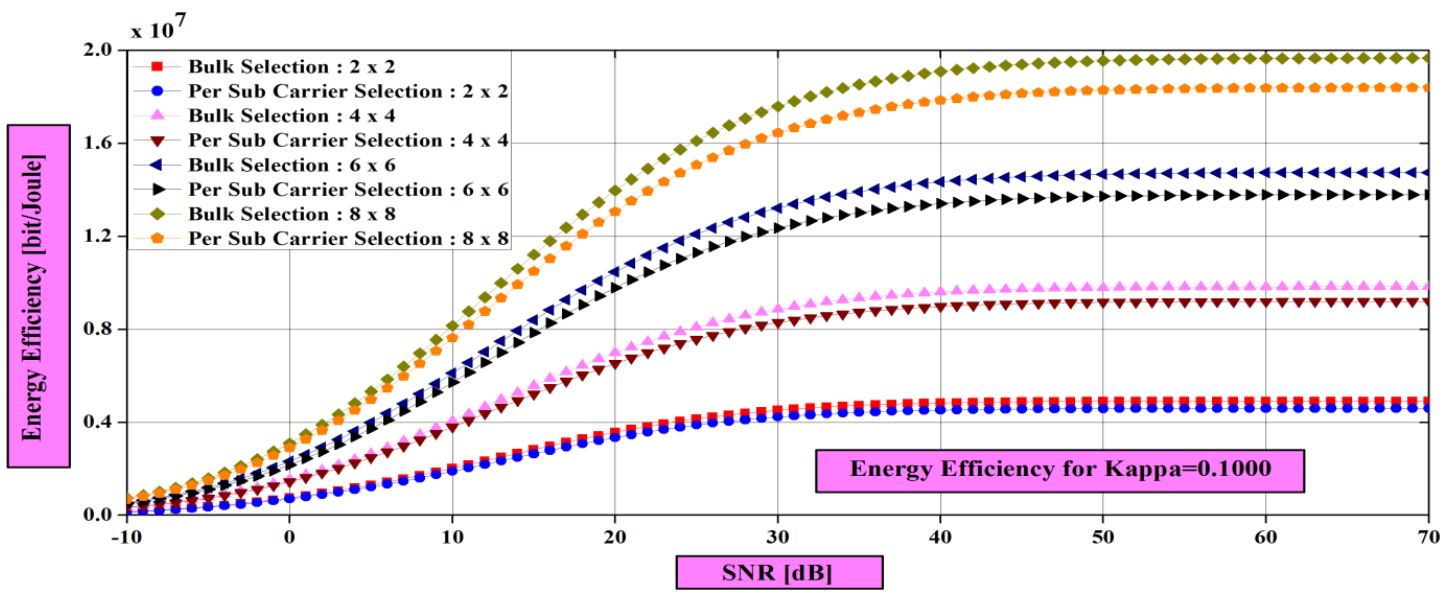

Figure 7. Energy Efficiency of MIMO-OFDM channel v/s SNR for Bulk and Per-subcarrier antenna selection techniques using various transmit and receive antennas schemes $(2 \times 2,4 \times 4,6 \times 6,8 \times 8)$ with hardware impairments (Kappa=0.1). 


\section{CONCLUSION}

In this paper, the Energy Efficiency or capacity of MIMO-OFDM channel for Bulk and Persubcarrier antenna selection techniques with or without hardware impairments $\{$ Kappa $\in(0.050 .1)\}$ is presented. The Energy Efficiency depends on many important factors such as the number of transmit and receive antennas, hardware impairments like transmit and receive power consumption etc., SNR and various antenna selection techniques. These hardware impairments limit the energy efficiency in the high SNR region as compared to the conventional one and energy efficiency decreases as the level of hardware impairments $\{k \in(0.05,0.1)\}$ increases. However, the energy efficiency increases with increase in the number of transmitting and receiving antenna but it increases the hardware cost. To meet the problem of hardware cost, it requires to use the various antenna selection techniques like Bulk and Per-subcarrier selection. Also, when we compared the Bulk and Per-subcarrier antenna selection techniques, it is observed that Bulk antenna selection has more energy efficiency than per subcarrier antenna selection in the high SNR region (20 to $70 \mathrm{~dB}$ ) since the per-subcarrier selection scheme requires a larger number of radio-frequency (RF) chains which increases the total power consumption of the system. But the energy efficiency remain almost same for both antenna selection techniques in the low SNR region (-10 to $20 \mathrm{~dB})$.

\section{REFERENCES}

[1] S. N. Raut and R. M. Jalnekar, "Performance Enhancement in SU and MU MIMO-OFDM Technique for Wireless Communication: A Review," International Journal of Electrical and Computer Engineering (IJECE), vol. 7, no. 5, pp. 2459-2467, Sep. 2017.

[2] J. Joung, C. K. Ho, K. Adachi, and S. Sun, "A survey on power-amplifier centric techniques for spectrum and energy efficient wireless communications," IEEE Communication Surveys Tutorial., vol. 17, no. 1, pp. 315-333, March 2014.

[3] N. B. Mehta, A. F. Molisch and S. Kashyap, "Antenna selection in LTE: From motivation to specification," IEEE Communication Magazine, vol. 50, no. 10,pp. 144-150, Oct. 2012.

[4] Abdul Latif, Moon Ho Lee, "Transmit Antenna Selection in the High SNR MIMO downlink correlated channel," Proceeding of IEEE on Information and Communication Technology Convergence (ICTC), pp. 220-224, 19-21Oct. 2016.

[5] Gaojie Chen, Yu Gong; Pei Xiao, Jonathon A. Chambers, "Dual Antenna Selection in secure cognitive radio networks, "IEEE Transaction on Vehicular Technology, vol. 65, no. 10,pp. 7993-8002, Oct. 2016.

[6] Kun Qian and Wen-Qin Wang, "Energy-Efficient Antenna Selection in green MIMO relaying communication Systems, ”IEEE Journal of Communications and Networks, vol. 18, no. 3,pp. 320-326, June 2016.

[7] Masoud Arash, Ehsan Yazdian, "Antenna Selection: A novel approach to improve Energy Efficiency in Massive MIMO systems,"Proceeding of IEEE on Computer and Knowledge Engineering (ICCKE), pp. 106-110, Oct. 2016.

[8] Pierluigi Vito Amadori, Christos Masouros, "Interference driven Antenna Selection for massive multi-user MIMO,"IEEE Transaction on Vehicular Technology, vol. 65, no. 8,pp. 5944-5958, Aug. 2016.

[9] Emil Björnson, Jakob Hoydis, Marios Kountouris, Mérouane Debbah, "Massive MIMO system with non-ideal hardware: Energy efficiency, estimation and capacity limits, "IEEE Transaction on Information Theory, vol. 60, no. 11,pp. 7112-7139, Sep. 2014.

[10] Ngoc Phuc Le, Farzad Safaei, Le Chung Tran, "Antenna selection strategies for MIMO-OFDM wireless systems: Energy Efficiency Perspective," IEEE Transaction on Vehicular Technology, vol. 65, no. 4,pp. 2048-2062, April. 2016.

[11] J. P. Coon and M. Sandell, "Combined bulk and per-tone transmit antenna selection in OFDM systems," IEEE Communication Letter, vol. 14, no.5,pp. 426-428, May 2010.

[12] Kehinde Odeyemi and Erastus Ogunti, "Capacity Enhancement for High Data Rate Wireless Communication System," International Journal of Electrical and Computer Engineering (IJECE), vol. 4, no. 5, pp. 800-809, Oct. 2014.

[13] Mir Muhammad Lodro and Muhammad Hanif, "Ergodic Capacity of MIMO Correlated Channels in Multipath Fading Environment with known Channel State Information," International Journal of Electrical and Computer Engineering (IJECE), vol. 2, no. 5, pp. 691-697, Sep. 2012.

[14] S. Sanayei and A. Nosratinia, "Capacity of MIMO channels with antenna selection," IEEE Trans. Inf. Theory, vol. 53, no. 11, pp. 4356-4362,Nov. 2007.

[15] Emil Bjornson, Per Zetterberg, Mats Bengtsson, Bjorn Ottersten, “ Capacity limits and multiplexing gains of MIMO channels with transceiver impairments, " IEEE Communications Letters, vol. 60, no. 11,pp. 7112-7139, Sep. 2014.

[16] C. Studer, M. Wenk, and A. Burg, "MIMO transmission with residual transmit-RF impairments," Proceeding of IEEE on WSA, 2010. 